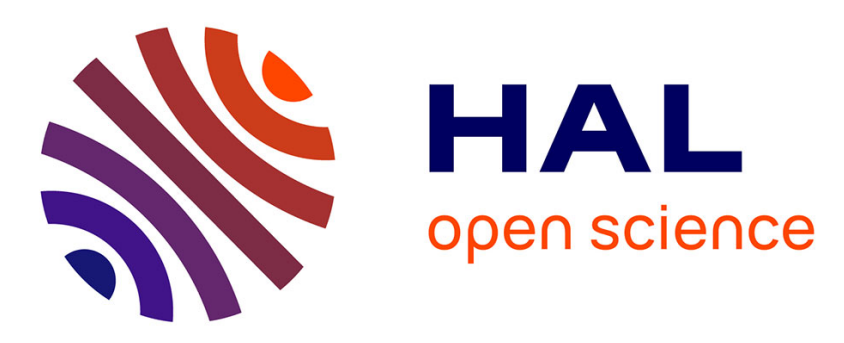

\title{
Probiotic from human breast milk, Lactobacillus fermentum, promotes growth in animal model of chronic malnutrition
}

Pierre Poinsot, Armelle Penhoat, Mélanie Mitchell, Valérie Sauvinet, Laure

Meiller, Corinne Louche-Pélissier, E. Meugnier, Mathias Ruiz, Martin

Schwarzer, Marie-Caroline Michalski, et al.

\section{To cite this version:}

Pierre Poinsot, Armelle Penhoat, Mélanie Mitchell, Valérie Sauvinet, Laure Meiller, et al.. Probiotic from human breast milk, Lactobacillus fermentum, promotes growth in animal model of chronic malnutrition. Pediatric Research, 2020, 88 (3), pp.374 - 381. 10.1038/s41390-020-0774-0 . hal-02998911

\section{HAL Id: hal-02998911 \\ https://hal.science/hal-02998911}

Submitted on 20 Nov 2020

HAL is a multi-disciplinary open access archive for the deposit and dissemination of scientific research documents, whether they are published or not. The documents may come from teaching and research institutions in France or abroad, or from public or private research centers.
L'archive ouverte pluridisciplinaire HAL, est destinée au dépôt et à la diffusion de documents scientifiques de niveau recherche, publiés ou non, émanant des établissements d'enseignement et de recherche français ou étrangers, des laboratoires publics ou privés. 


\section{BASIC SCIENCE ARTICLE Probiotic from human breast milk, Lactobacillus fermentum, promotes growth in animal model of chronic malnutrition}

Pierre Poinsot ${ }^{1,2,3}$, Armelle Penhoat ${ }^{2}$, Mélanie Mitchell ${ }^{1}$, Valérie Sauvinet ${ }^{2,4}$, Laure Meiller ${ }^{2,4}$, Corinne Louche-Pélissier ${ }^{4}$, Emmanuelle Meugnier ${ }^{2}$, Mathias Ruiz ${ }^{2,3}$, Martin Schwarzer ${ }^{1,5}$, Marie-Caroline Michalski ${ }^{2}$, François Leulier ${ }^{1}$ and Noël Peretti ${ }^{2,3}$

BACKGROUND: Chronic undernutrition leads to growth hormone resistance and poor growth in children, which has been shown to be modulated by microbiota. We studied whether Lactobacillus fermentum CECT5716 (Lf CECT5716), isolated from mother's breast milk, could promote juvenile growth through the modulation of lipid absorption in a model of starvation.

METHODS: Germ-free (GF) Drosophila melanogaster larvae were inoculated with $L f$ CECT5716 in conditions of undernutrition with and

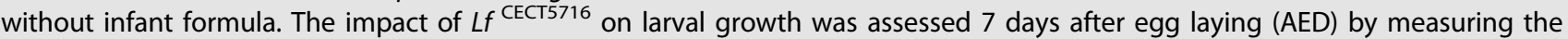
larval size and on maturation by measuring the emergence of pupae during 21 days AED. For lipid absorption test, Caco2/TC7 intestinal cells were incubated with $L f^{\text {CECT5716 }}$ and challenged with mixed lipid micelles.

RESULTS: The mono-associated larvae with $L f$ CECT5716 were significantly longer than GF larvae $(3.7$ vs $2.5 \mathrm{~mm} ; p<0.0001)$. The effect was maintained when $L f^{C E C T 5716}$ was added to the infant formula. The maturation time of larvae was accelerated by $L f$ CECT5716 (12 vs 13.2 days; $p=0.01$ ). Lf ${ }^{\text {CECT5716 }}$ did not have significant impact on lipid absorption in Caco2/TC7 cells.

CONCLUSIONS: $L f{ }^{\text {CECT5716 }}$ is a growth-promoting strain upon undernutrition in Drosophila, with a maintained effect when added to an infant formula but without effect on lipid absorption in vitro.

Pediatric Research _\#\#\#\#\#\#\#\#\#\#\#\#\#\#\#\#\#\#_; https://doi.org/10.1038/s41390-020-0774-0

\section{INTRODUCTION}

According to a 2018 Food and Agriculture Organization/World Health Organization report, stunting affects 151 million children worldwide, which represented $50 \%$ of global child deaths at the time. ${ }^{1}$ Besides mortality, chronic undernutrition can severely impact child growth. After an early loss of weight, a decrease of the systemic growth rate due to a state of growth hormone resistance leads to poor height-for-age in children (stunting). ${ }^{2,3}$ The recovery of an optimal protein and energy intake can help these children to restore optimal growth, weight gain, and neuropsychological development and to reduce co-morbidity.

Gut microbiota profiles in undernourished children demonstrates several specific modifications. In a pediatric cohort from Bangladesh, Subramanian et al. found a stunting profile of gut microbiota, characterized by its relative immaturity. ${ }^{4}$ Recently, the capacity of the gut microbiota to change juvenile growth has been described as a complex interaction between the nutritional environment, the genetic background, and the bacterial phyla. ${ }^{5}$ Moreover, in murine models, the gut microbiota appears to be essential to maintain juvenile growth and optimal function of the somatotropic axis. ${ }^{6}$ However, it is still unclear how this undernutrition-altered microbiota influences the growth of its host. Two studies have transplanted the gut microbiota from stunted and healthy children to GF mice. ${ }^{7,8}$ In the same nutritional environment, the stunted microbiota induced more weight loss than microbiota from the healthy children. Interestingly, the beneficial effect of a healthy microbiota could be restored by colonization with two specific selected strains found to be enriched in the normal weight children (Rhuminococcus gnavus and Clostridium symbosium). ${ }^{8}$ Furthermore, Schwarzer et al. have shown that specific isolates of Lactobacillus plantarum could maintain mouse juvenile growth and the somatotropic axis in conditions of undernutrition. ${ }^{6}$ All these results suggest that a complete gut microbiota or some selected strains are able to induce major changes in the nutritional phenotype of its host. Given the ease of cultivating lactobacilli, functional strains from these phyla are appealing candidates for clinical studies. With this prospect, the application of lactobacilli strains to restore juvenile growth in animals and humans with undernutrition was patented in 2015 (ref. patent: WO2015173386A1).

In previous publications, the lactobacilli studied by Schwarzer et al. were identified and functionally validated in monoassociation in drosophila. ${ }^{9}$ Germ-free (GF) Drosophila melanogaster is a powerful model to study host-microbiota interactions. Drosophila presents a microbiota shaped by its nutritional environment and shares several functions with the human microbiota. Lactobacilli have a high prevalence in the microbiome of the fly. ${ }^{10,11}$ This indicates that the interaction model Drosophila-Lactobacillus is a powerful model to test clinically approved bacterial strains to improve infantile-stunting phenotypes and build new nutritional strategies in humans.

With this in mind, we studied Lactobacillus fermentum CECT5716 $\left(L f^{\mathrm{CECT5716}}\right)$. This strain has been isolated from healthy mother's breast milk and has potential probiotic properties. ${ }^{12}$ Indeed,

\footnotetext{
${ }^{1}$ Institut de Génomique Fonctionnelle de Lyon, Université de Lyon, Ecole Normale Supérieure de Lyon, CNRS UMR5242, UCBL1, Lyon, France; ${ }^{2}$ Univ Lyon, CarMeN Laboratory, INSERM, INRA, INSA Lyon, Université Claude Bernard Lyon 1, 69310 Pierre-Benite, France; ${ }^{3}$ Department of Pediatric Nutrition, Hôpital Femme Mère Enfant, Univ Lyon, Hospice Civil

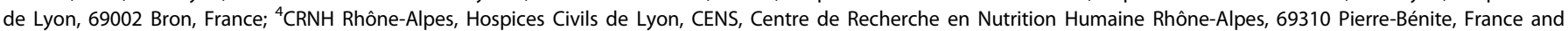
${ }^{5}$ Laboratory of Gnotobiology, Institute of Microbiology of the Czech Academy of Sciences, Novy Hradek, Czech Republic

Correspondence: Pierre Poinsot (pierre.poinsot@chu-lyon.fr)
}

Received: 17 September 2019 Revised: 3 January 2020 Accepted: 15 January 2020

Published online: 05 February 2020 
Lf CECT5716 has demonstrated interesting capacities in immunemodulation, prevents bacterial and viral infections, colitis lesions in mice and oxidative stress, and has an antihypertensive effect. $^{13,14}$ The strain is already used in an infant milk formula for healthy children. ${ }^{15}$ All these parameters make $L f{ }^{\text {CECT5716 a }}$ good candidate for testing its potential growth-promoting effect in animal models. We therefore used the drosophila model to investigate the impact of $L f^{\mathrm{CECT5716}}$ on two different parameters, juvenile growth and maturation time.

Because the somatotropic axis could be influenced by dietary lipids as a caloric source, we used Caco2/TC7 cells, derived from a human colorectal carcinoma that is spontaneously highly differentiated, to study the impact of $L f^{\text {CECT5716 }}$ on lipid uptake. ${ }^{16,17}$

The aims of this work were: (1) to study whether $L f$ CECT5716 could promote juvenile growth and maturation in a GF drosophila model upon undernutrition and to compare this effect with two other calibrating strains, (2) to determine whether the promoting effect is maintained when $L f^{\text {CECT5716 }}$ is added to infant formula and whether there is a dose-dependent effect, and (3) to evaluate whether $L f$ CECT5716 could increase lipid absorption on a cocultured model of enterocytes, a hypothesis susceptible to explain the growth-promoting effect of $L f^{\mathrm{CECT} 5716}$ on juvenile growth.

\section{METHODS}

Impact of $L f$ CECT5716 on animal growth

GF flies. Yellow white Drosophila $(y w)$ was used as the reference strain. Drosophilas were bred at $25^{\circ} \mathrm{C}$ in a light cycle incubator on an optimal yeast/cornmeal medium (Table S1). The conventional microbiota in drosophila was removed by bleaching and cultivating the embryos on an autoclaved media. GF stocks were routinely maintained on an optimal diet supplemented with antibiotics $(50 \mu \mathrm{g}$ ampicillin, $50 \mu \mathrm{g}$ tetracycline, $50 \mu \mathrm{g}$ kanamycin, and $15 \mu \mathrm{g}$ erythromycin). Experimental GF animals were maintained on an autoclaved diet without antibiotics. Chronic undernutrition was induced by decreasing the yeast content, the main protein source in the diet (Table S1).

Bacterial strains. Lf ${ }^{\text {CECT5716 }}$ isolated from human breast milk was provided by LACTALIS NUTRITION ${ }^{\oplus}$, Spain in lyophilized form $\left(10^{11}\right.$ colony-forming units (CFU)/g). Lactobacillus plantarum NIZO2877 $\left(L p^{\mathrm{Nizo2877}}\right.$ ) provided by the NIZO organization (The Netherlands) and Lactobacillus plantarum WJL ( $L p^{\mathrm{WJL}}$ ) isolated from laboratoryraised flies in Professor Won-jae Lee's laboratory (Seoul National University, South Korea) were used as calibrating strains. ${ }^{18}$ The $L p^{\text {NIZO2877 }}$ effect on drosophila juvenile growth have been defined as intermediate, whereas $L p{ }^{\text {WJL }}$ have been defined as a marked growth-promoting strain on drosophila juvenile growth in previous experiments (data not published).

Bacterial and infant formula inoculum preparation. On the day before the experiments, $L p{ }^{\mathrm{NIZO} 2877}$ and $L p{ }^{\mathrm{WJL}}$ were cultured overnight at $37^{\circ} \mathrm{C}$ in Man, Rogosa, and Sharpe broth (MRS) culture medium. The following day, optical density (OD) was measured at $600 \mathrm{~nm}$ to determine the number of CFU/mL. After centrifugation, the supernatant was removed and the pellet was resuspended in a sterile phosphate-buffered saline (PBS) solution and inoculated in appropriate concentration for the experiment. Lf CECT5716 (10 ${ }^{11}$ CFU/g), a ready-to-use hydrolyzed milk formula containing the $L f$ CECT5716 in $10^{6}$ CFU/g (HMF-LF4), and a sterile hydrolyzed milk formula (SHMF) were obtained under lyophilized form. Infant formulas were dissolved in PBS for a final standard osmolarity. HMF and HMF-LF4 nutritional compositions are shown in Table S2.

Juvenile growth test: larval size protocol. This protocol was performed with a low yeast diet for a chronic undernutrition condition.

The day before inoculation of embryos (considered as Day -1), axenic $y w$ drosophila were transferred to an egg-laying cage containing an optimal diet medium with antibiotics. In the evening, optimal diet caps were changed for low yeast diet medium without antibiotics (Table S1). On Day 0, overnight egg-laying caps were collected. A thin slice of medium containing approximately 40 eggs was cut with a sterile scalpel (using a binocular) and placed on a new medium cap with low yeast diet. This step was repeated in triplicates for each experimental condition (total of 120 individuals per condition). Each batch of 40 eggs was inoculated with: sterile PBS (GF condition) or HMF or $10^{8}$ CFU (for $L p^{\text {WJL }}, L p^{\text {NIZO2877, }}, L f^{\text {CECT5716) }}$ or HMF-LF4 at $10^{4}$ CFU. A third homemade formula was used adding Lf CECT5716 into SHMF in order to obtain an inoculation at $10^{8}$ CFU (HMF-LF8). Larvae were then incubated for 7 days at $25^{\circ} \mathrm{C}$.

At Day 7, 60 larvae per condition were collected from the pooled triplicate caps on a microscopic slide, heat killed on a heating plate for $5 \mathrm{~s}$ at $100{ }^{\circ} \mathrm{C}$, and put under a cover slide in a $80 \%$ glycerol solution. $\mathrm{A}$ picture of each slide was taken with a Leica ${ }^{\circledR}$ M205FA binocular coupled with a Leica ${ }^{\circledR}$ DFC450 camera. Pictures were exported by the Leica Application Suite ${ }^{\odot}$ software. Longitudinal larval size was then quantified with the ImageJ software (National Institutes of Health).

Maturation time test: developmental timing protocol. The same conditions were used for this experiment as with juvenile growth, except the HMF-LF4. Experiments were carried out on a low yeast diet. On Day -1 , axenic yw drosophila were transferred to an egglaying cage as described previously. On Day 0, overnight egglaying caps were collected. A thin slice of medium containing exactly 40 eggs was cut with a sterilized scalpel (using a binocular) and placed on new medium tubes with a low yeast medium. This step was repeated in triplicate for each experimental condition. Each tube was inoculated with $10^{8} \mathrm{CFU}$ or its equivalent dilution for the infant formula (prepared as described previously). Tubes were incubated for 21 days at $25^{\circ} \mathrm{C}$ and pupae emergence was monitored each day. Developmental timing was expressed by the median duration of the $50 \%$ point of adult emergence $\left(D_{50}\right)$. This experiment was done in triplicates twice for each experimental condition.

Impact of $L f$ CECT5716 on lipid absorption

Bacterial strain. Lf ${ }^{\text {CECT5716 }}$ was isolated from the lyophilized form on an MRS agar culture plate left for $72 \mathrm{~h}$ at $37^{\circ} \mathrm{C}$. One isolated CFU was inoculated in MRS medium and incubated at $37^{\circ} \mathrm{C}$ for $18 \mathrm{~h}$. The resulting culture was stored at $-80^{\circ} \mathrm{C}$ with $20 \%$ glycerol. The day of the experiment, OD was measured at $600 \mathrm{~nm}$ to determine $\mathrm{CFU} / \mathrm{mL}$. Appropriate volumes of bacterial cultures were centrifuged and the pellet was suspended in Dulbecco's modified Eagle medium (DMEM) for appropriate multiplicity of infection (MOI) ratio. Three MOls were used for this protocol: 1 bacterium for 1 cell, 10 bacteria for 1 cell, and 100 bacteria for 1 cell.

Caco2/TC7 cells. Caco2/TC7 cells were grown in $75 \mathrm{~cm}^{2}$ flasks at $37^{\circ} \mathrm{C}$ and $10 \% \mathrm{CO}_{2}$ in DMEM Glutamax ${ }^{\mathrm{TM}}$ without pyruvate containing $4.5 \mathrm{~g} / \mathrm{L}$ glucose, $1 \%$ non-essential amino acids, and $1 \%$ antibiotics (GIBCO ${ }^{\circ}$, ThermoFischer Scientific ${ }^{\text {TM }}$, USA) and supplemented with $20 \%$ heat-inactivated fetal calf serum (FCS, PAA). Cultures were split when they reached $80 \%$ confluence, using trypsin-EDTA. For experiments, Caco-2/TC7 intestinal cells were seeded into 6-well plates $\left(5 \times 10^{4}\right.$ cells $\left./ \mathrm{cm}^{2}\right)$ on Transwell filter inserts $(0.4-\mu \mathrm{m}$ pore size Polyester Membrane, Costar, Cambridge, MA, USA) to reproduce the intestinal barrier. Cells were grown to confluence in complete medium for 1 week. Cells were then cultured in asymmetric conditions, with medium containing FCS in the basal compartment and serum-free medium in the apical compartment until total differentiation (around 21 days after seeding). The medium was changed every 2-3 days. During culture, the trans-epithelial electrical resistance was assessed using Millicell-ERS apparatus (Millipore, Bedford, MA). ${ }^{19}$ 
Mixed lipid micelles. To study lipid absorption and secretion by intestinal cells, Caco-2/TC7 cells were incubated with so-called "mixed lipid micelles," which simulated postprandial lipid structures in the intestinal lumen and contained $\left[\mathrm{U}-{ }^{13} \mathrm{C}\right]$ oleic acid $(0.5 \mathrm{mM}$; a stable isotope tracer for gold-standard fatty acid absorption tests), 2-oleylglycerol $(0.2 \mathrm{mM})$, phosphatidylcholine $(0.4 \mathrm{mM})$, lysophosphatidylcholine $(0.2 \mathrm{mM})$, cholesterol $(0.05 \mathrm{mM})$, and taurocholate $(2 \mathrm{mM})$. The final solution was vortexed in DMEM Glutamax medium and further dispersed in an ultrasonic bath for $5 \mathrm{~min}$ at room temperature. ${ }^{16}$

Lipid uptake assay. On the day before the experiment, Caco2/TC7 layers were washed two times with DMEM. Apical and baso-lateral media were changed for clean DMEM without FCS to put cells into a fasting state. On the day of the experiment, Lf CECT5716 was inoculated in $1.5 \mathrm{~mL}$ of serum-free DMEM without antibiotics in triplicate for each MOI for $6 \mathrm{~h}$. GF control was done at the same time with DMEM only. After $6 \mathrm{~h}$ of bacterial incubation, the apical supernatant was removed and the apical chamber was washed 4 times with $1 \mathrm{~mL}$ of DMEM to leave only adherent bacteria. Then $1.5 \mathrm{~mL}$ of serum-free culture medium containing micelles of $\left[\mathrm{U}-{ }^{13} \mathrm{C}\right]$ oleic acid ([U- $\left.{ }^{13} \mathrm{C}\right] \mathrm{C}_{18: 1}, 0.5 \mathrm{mM}$ ) (EURISOTOP, Saint Aubin, France) prepared as described previously was added to the apical chamber, and cells were incubated at $37^{\circ} \mathrm{C}$ and $10 \% \mathrm{CO}_{2}$. After $18 \mathrm{~h}$ of incubation, apical media, basal media, and samples from mixed bile salt micelles were collected and stored at $-20^{\circ} \mathrm{C}$. Then the monolayers were washed 4 times by ice-cold PBS, harvested, and stored in dry tubes at $-80^{\circ} \mathrm{C}$. Cells were then resuspended in $500 \mu \mathrm{L}$ of hexane and a mechanical lysis was performed using a pestle. After addition of an internal standard (C17:0, heptadecanoic acid, $22 \mu \mathrm{L}, 0.25 \mathrm{mM}$ for basal; $90 \mu \mathrm{L}, 1 \mathrm{mM}$ for apical; $115 \mu \mathrm{L}, 2.6 \mathrm{mM}$ for cells; and $125 \mu \mathrm{L}, 1 \mathrm{mM}$ for micellar medias), total lipids from $750 \mu \mathrm{L}$ basal, $330 \mu \mathrm{L}$ apical, the entire lysate, or $250 \mu \mathrm{L}$ micellar medias were directly methylated using acetyl chloride and the remaining amount of ${ }^{13} \mathrm{C}$-oleic acid in both media was quantified by gas chromatography-mass spectrometry (GC-MS; electron ionization, scan-sim mode) using a quadrupole mass spectrometer (model MS 5975, Agilent Technologies, Massy, France) connected to a gas chromatograph (model GC6890, Agilent Technologies)) as previously described. $^{20}$ It was equipped with a fused silica column (SP-2380, $30 \mathrm{~m} \times 0.25 \mathrm{~mm} \times 0.20 \mu \mathrm{m}$ film thickness; Supelco). Helium was used as the carrier gas. Injection $(1 \mu \mathrm{L})$ was performed in splitless mode at $250^{\circ} \mathrm{C}$. Oleic acid was separated at constant flow $(1.2 \mathrm{~mL} / \mathrm{min})$ with the following oven program: (a) $50^{\circ} \mathrm{C}$ for $1 \mathrm{~min}$; (b) increase at a rate of $11^{\circ} \mathrm{C} / \mathrm{min}$ to $175^{\circ} \mathrm{C}$ during $5.64 \mathrm{~min}$; (c) increase at a rate of $5^{\circ} \mathrm{C} /$ min to $220^{\circ} \mathrm{C}$; (d) post-run $245^{\circ} \mathrm{C}$ for $1 \mathrm{~min}$. Total ion chromatogram was used for quantification over a mass range of $\mathrm{m} / \mathrm{z} 35-450$. Selected ion monitoring was employed, with targeted ion $\mathrm{m} / \mathrm{z} 296$ and 314 for the natural and $\left[U^{13} \mathrm{C}\right]$ Oleate, respectively, for enrichment measurement. $^{20}$

Caco2/TC7 cell RNA extraction and reverse transcription-quantitative $P C R(R T-q P C R)$. The experiment described previously was reproduced for the RNA extraction. In the same way, oleic acid content in apical media was measured by GC-MS to confirm the lack of effect of $L f^{\text {CECT5716 }}$ on lipid uptake. Then Caco2/TC7 cells were washed 4 times by ice-cold PBS. Monolayers were harvested and stored in dry tubes at $-80^{\circ} \mathrm{C}$. Total RNA was extracted from the Caco2/TC7 monolayer with TRI Reagent (Sigma, Saint-Quentin-Fallavier, France). RNA concentration was measured with Multiskan GO microplate spectrophotometer (ThermoFischer Scientific, Waltham, MA, USA), and samples with A260/280 ratios between 1.7 and 2.1 were considered of good purity. RT was performed using the PrimeScript RT Reagent Kit (Ozyme, Saint Quentin en Yvelines, France) with $1 \mu \mathrm{g}$ of RNA. RT-qPCR assays were performed using Rotor-Gene $Q$ (Qiagen, Hilden, Germany) and SYBR qPCR Premix Ex Taq (Tli a

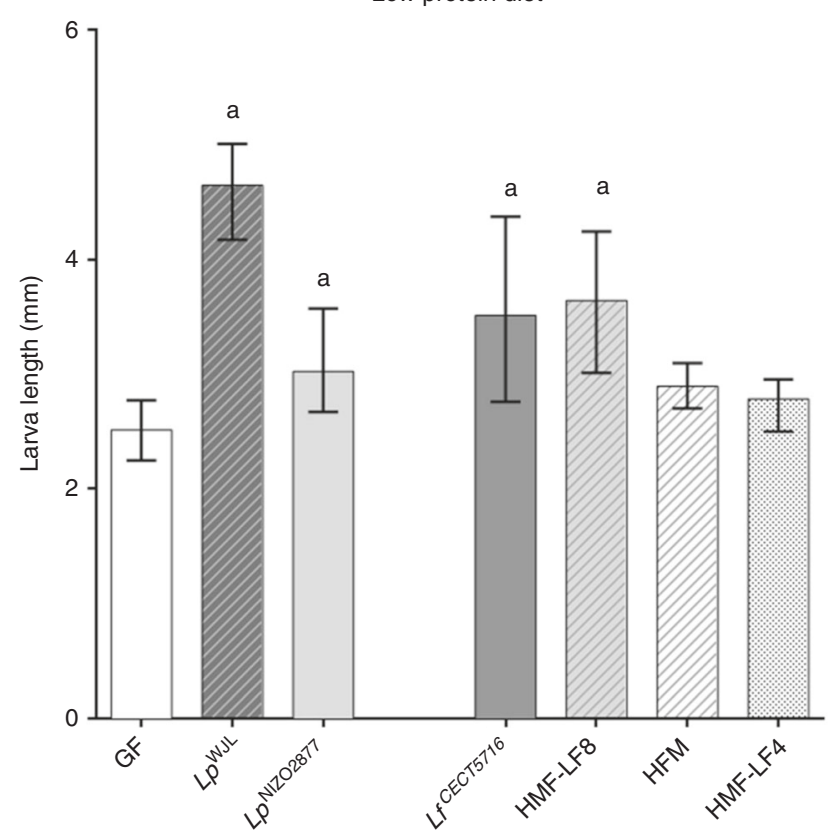

b

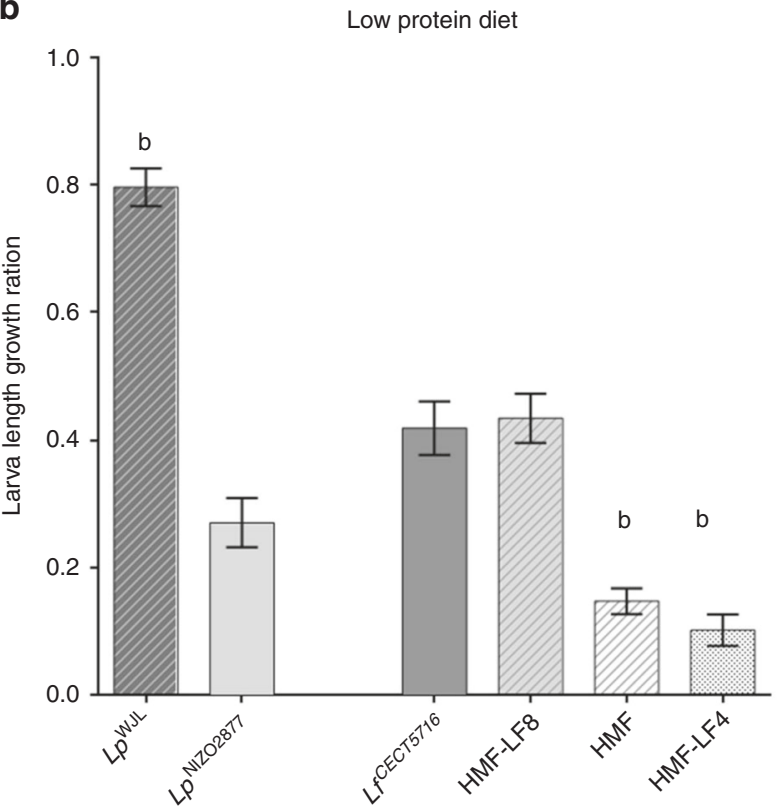

Fig. 1 Impact of $L f$ CECT5716 on drosophila juvenile growth after 7 days of incubation. The growth-promoting effect of $L f$ CECT5716 was assessed after 7 days of mono-association with drosophila yellow white-type larvae on a low-protein diet. The median larval survival rate was $96.5 \%$ for Lp WJL, $96 \%$ for Lp NIZO2877 and $91 \%$ for Lf CECT5716. a The growth-promoting effect was compared with two control strains: $L p$ NIZO2877 $\left(3.33 \times 10^{8} \mathrm{CFU} / \mathrm{mL}\right.$ of inoculum, qualified by its intermediate effect) and $L p$ WJL $\left(3.33 \times 10^{8} \mathrm{CFU} / \mathrm{mL}\right.$, qualified by its marked effect). The growth-promoting effect of $L f$ CECT5716 was assessed in three infant formulas: HMF-LF4 (containing $3.33 \times 10^{4} \mathrm{CFU} / \mathrm{mL}$ in inoculum), HMF-LF8 (containing $3.33 \times$ $10^{8} \mathrm{CFU} / \mathrm{mL}$ in inoculum), and HMF. A statistical difference compared to GF was defined by $p<0.001$ on Kruskal-Wallis test (a). b The growth ratio of the different mono-associations and formulas was normalized on GF condition and compared to the Lf CECT5716 growth ratio with a statistical difference defined by $p<0.001$ on Kruskal-Wallis test (b). 
RNaseH Plus) reagents. The list of the PCR primers used is shown in Table S3. Tata-box-binding protein expression was used as internal standard for normalization of target mRNA expression.

\section{Statistics}

Larval sizes and $D_{50}$ were expressed by their median according to their rank. Kruskal-Wallis test was used to compare the different experimental groups due to a non-normal distribution of length values. A statistical difference between larval lengths was defined by $p<0.001$ on Kruskal-Wallis test comparison. A statistical difference between $D_{50}$ was defined by $p<0.05$ on Kruskal-Wallis test comparison. For the lipid absorption assay on Caco2/TC7 cells, a statistical difference was determined by $p<0.05$ on oneway analysis of variance. Multiple comparisons were done with using Prism Graphpad ${ }^{\oplus}$ v6.

The larval growth ratio was defined as:

$\underline{\text { [(Larval length mono-associated) -(Median larval length GF)] }}$

$$
\text { Median larval length GF }
$$

Growth ratios were expressed by their median according to their interquartile range. A statistical difference was defined by $p<0.05$ on Kruskal-Wallis test comparison.

\section{RESULTS}

Impact of $\angle f^{C E C T 5716}$ on drosophila juvenile growth and maturation upon undernutrition

Impact of Lf ${ }^{\text {CECT5716 }}$ on juvenile growth while consuming a low yeast diet and comparison with other bacterial strains. Under low yeast diet conditions, Lf ${ }^{\text {CECT5716 }}$ had a significant growth-promoting effect on drosophila larvae. GF larvae were statistically shorter $(2.5 \mathrm{~mm} ; 1.4-4)$ than larvae mono-associated with $L f$ CECT5716 (3.7 mm; 2.2-5.4; $p<0.0001$ ) (Fig. 1a). The specific intensity of the growth-promoting effect of $L f f^{\text {CECT5716 }}$ was compared to two other strains, namely, $L p^{\mathrm{NIZO} 2877}$ and $L p^{\mathrm{WJL}}$. Median larval size of monoassociation with $L p{ }^{\mathrm{NIZO} 2877}(3 \mathrm{~mm} ; 1.8-5.5)$ and $L p$ WJL $(4.6 \mathrm{~mm}$; 3.2-4.7) were statistically higher compared to the GF larvae $(2.5 \mathrm{~mm} ; 1.4-4 ; p<0.0001)$. These results confirmed the growthpromoting ability of the control strains. Compared to these two strains, the median larval size of the $L f^{\text {CECT5716 }}$ mono-associated larvae was significantly different and lower only when compared to the $L p{ }^{\text {WJL }}$ mono-association ( $p<0.0001$; Fig. 1 a). Indeed, the growth-promoting effects of $L f{ }^{\text {CECT5716 }}$ and $L p{ }^{\text {NIZO2877 }}$ were not statistically different. These results support a species- and straindependent effect of the different bacteria on juvenile growth in drosophila, with $L p$ NIZO2877 and $L f$ CECT5716 presenting an intermediate functionality on juvenile growth, while $L p$ WJL

a
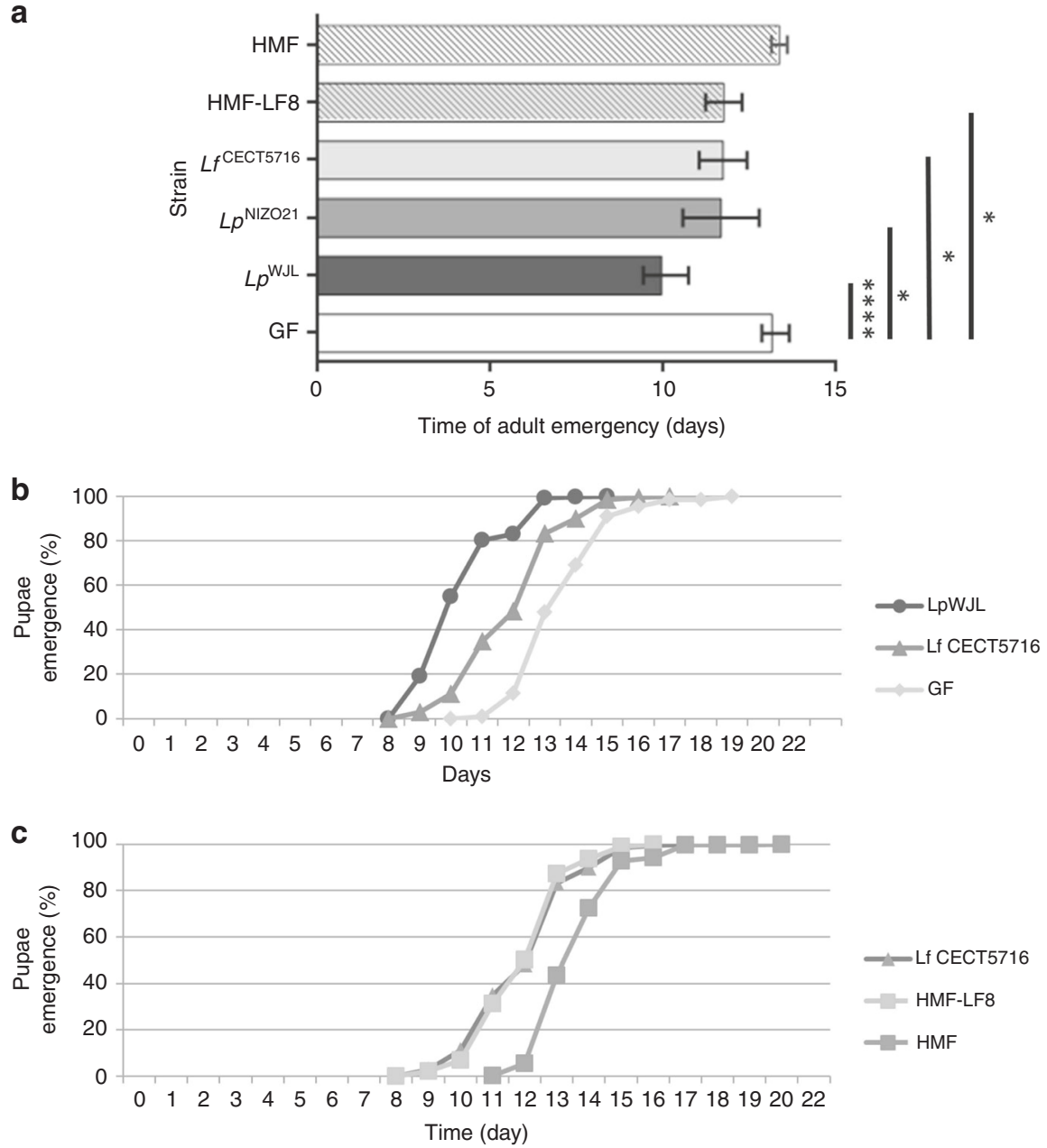

Fig. 2 Impact of Lf CECT5716 on larvae maturation time after 21 days of incubation on a low yeast diet. GF yellow white-type drosophila eggs were incubated 21 days with $L f{ }^{\text {CECT5716 }}\left(3.33 \times 10^{8} \mathrm{CFU} / \mathrm{mL}\right), L p$ NIZO2877 $\left(3.33 \times 10^{8} \mathrm{CFU} / \mathrm{mL}\right), L p$ WJL $\left(3.33 \times 10^{8} \mathrm{CFU} / \mathrm{mL}\right)$, HMF-LF8 $\left(3.33 \times 10^{8} \mathrm{CFU} / \mathrm{mL}\right)$, and HMF. a The $\mathrm{D}_{50}$ was assessed for each condition on a low yeast diet by counting the pupae each day. b, c The time delay was also calculated for each condition on a low yeast diet. 


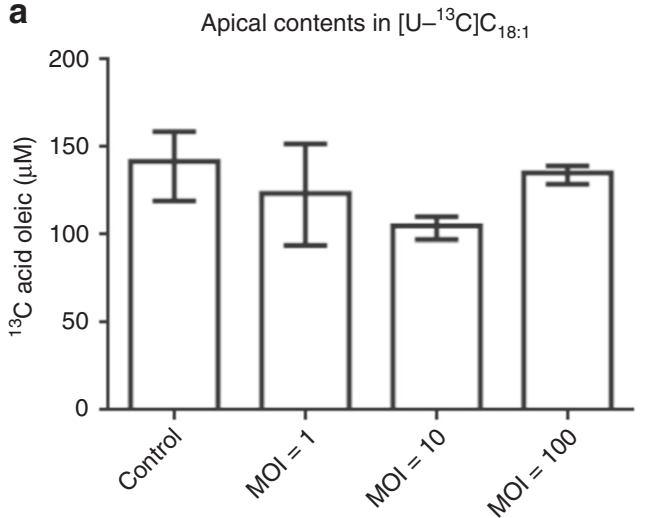

Multiplicity of infection

b

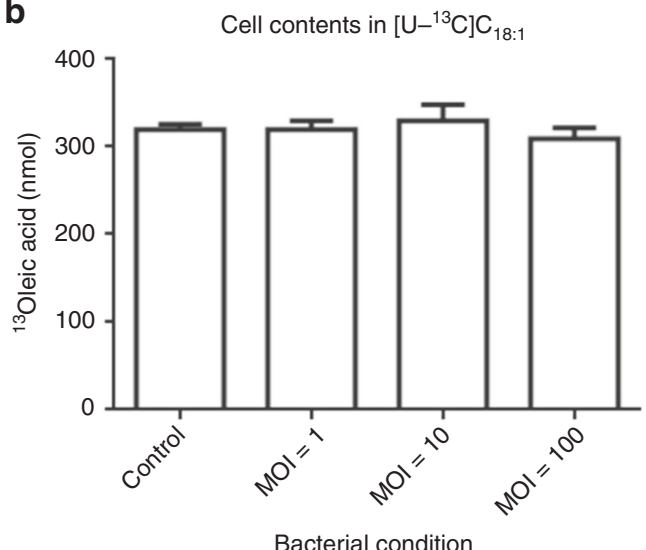

Fig. 3 Impact of $L f{ }^{\mathrm{CECT5716}}$ on $\left[\mathrm{U}^{13} \mathrm{C}\right] \mathrm{C}_{18: 1}$ (oleic acid) uptake in Caco2/TC7 cells. After $6 \mathrm{~h}$ of co-incubation between Lf CECT5716 and Caco2/TC7 monolayer, mixed lipid micelles were incubated during $16 \mathrm{~h}$ in the apical chamber. A sterile control and 3 $\mathrm{MOI}\left(1,10\right.$, and 100) were used for the experiment. [U $\left.{ }^{13} \mathrm{C}\right] \mathrm{C}_{18: 1}$ content was measured in $\mathbf{a}$ apical medium and $\mathbf{b}$ cells by GC-MS. Experiment was done three times in triplicate.

presented a marked functionality. For all conditions, the median larval survival rate was over $90 \%$.

When tested alone, the HMF did not have any significant effect on larval size (2.9 mm; $1.6-3)$ compared to the GF larvae $(2.5 \mathrm{~mm}$; 1.4-4; $p=0.005)$. Similarly, the ready-to-use HMF-LF4 containing only $10^{4}$ CFU (2.8 mm; 1.6-4.4) did not have a significant effect on larval growth compared to the GF larvae $(2.5 \mathrm{~mm} ; 1.4-4 ; p=0.2)$ (Fig. 1a). However, when $10^{8}$ CFU of $L f^{\text {CECT5716 }}$ was added to the HMF (HMF-LF8), a significant growth-promoting effect was measured compared to the GF larvae $((3.6 \mathrm{~mm} ; 1.9-5)$ vs $(2.5 \mathrm{~mm}$; $1.4-4) p<0.0001)$. Moreover, this effect was statistically stronger compared to the HMF-LF4 $(2.9 \mathrm{~mm} ; 1.6-4.4 ; p<0.0001)$ larvae and to the HMF $(2.9 \mathrm{~mm} ; 1.6-4.3 ; p=0.0001)$. These results suggest that $L f$ CECT5716 is able to promote juvenile growth in drosophila larvae upon undernutrition but only when present above a critical inoculum concentration (superior to $10^{4} \mathrm{CFU}$ ).

To precise the power of the growth-promoting effect, we calculated the growth ratio of each strain and formula according to the GF condition (Fig. 1b). Interestingly, the $L p{ }^{\text {WJL }}$ had a statically higher larval growth ratio compared to $L f$ CECT5716 ((0.84; -0.05 to 1.26$)$ vs $(0.39 ;-0.13$ to 1.15$) ; p<0.0001))$. As expected, the Lp NIZO2877 and the HMF-LF8 had an equivalent growthpromoting effect compared to $L f^{\text {CECT5716 }}$ alone. Finally, HMF (0.15; -0.37 to 0.7$)$ and HMF-LF4 $(0.10 ;-0.36$ to 0.76$)$ had a significant lower effect than $L f$ CECT5716 alone. All these results confirm the promoting effect of $L f^{\mathrm{CECT5716}}$ alone or added in an infant formula.
Impact of the $\mathrm{Lf}{ }^{\mathrm{CECT} 5716}$ on the maturation time while on a low yeast diet and comparison with other strains of probiotics. Under a low yeast diet condition, the maturation time (emergence of the adult form) of larvae evaluated by the $D_{50}$ was statistically shorter by 1.2 days for $L f$ CECT5716 mono-associations compared to the GF condition ( $p=0.01$; Fig. $2 \mathrm{a}$, b). Similarly, the $D_{50}$ of $L p^{\mathrm{NIZO} 2877}$ and $L p{ }^{\mathrm{WJL}}$ mono-associations were statistically shorter compared to GF $(p<0.05$; Fig. 2a). Compared to $\mathrm{GF}$, the pupae emergence was shortened by 1.5 and 3.2 days for $L p$ NIzO2877 and $L p$ WJL, respectively. In the HMF condition, the $D_{50}$ was not statistically different from the GF larvae $(p>0.9)$. However, the addition of $L f^{\text {CECT5716 }}$ to the HMF (HMF-LF8) significantly shortened the pupae emergence by 1.6 days (( 12 days; $11-12.5)$ vs (13.2 days; $13-13.8)$; $p<0.05$; Fig. 2c). Moreover, the $D_{50}$ of the Lf CECT5716 monoassociation was not statistically different from the $D_{50}$ of the HMFLF8 condition $(p>0.9)$. These results show that, upon undernutrition, $L f$ CECT5716 is able to restore part of the developmental timing delay observed in drosophila larvae, in mono-association as well as when added to the HMF formula (HMF-LF8).

Impact of the $L f^{\text {CECT5716 }}$ on lipid uptake and gene regulation in Caco2/TC7

After incubation of $\mathrm{Caco} 2 / \mathrm{TC7}$ cells with mixed lipid micelles, there was no statistical difference of $U-{ }^{13} \mathrm{C}$ oleic acid content in the apical chamber or in the cell content between control and all experimental conditions with different probiotic concentrations (MOI; Fig. 3). Despite an increasing MOI, Lf ${ }^{\text {CECT5716 }}$ does not seem to have any specific effect on free fatty acid metabolism in enterocytes compared to GF condition in vitro. The major part of $\left[\mathrm{U}-{ }^{13} \mathrm{C}\right] \mathrm{C}_{18: 1}$ used by cells was $80 \%$ of $\left[\mathrm{U}-{ }^{13} \mathrm{C}\right] \mathrm{C}_{18: 1}$ mixed lipid micelle content. The mean $\left[\mathrm{U}^{13}{ }^{13} \mathrm{C}\right] \mathrm{C}_{18: 1}$ cell content was calculated and represented $79 \%$ of the $\left[\mathrm{U}^{-{ }^{13}} \mathrm{C}\right] \mathrm{C}_{18: 1}$ mixed lipid micelle content.

Moreover, the fold changes of the mRNA of $A P O-B, S A R 1 B$, and $M T T P$, the main genes involved in the regulation of chylomicron (CM) synthesis by enterocytes, appeared to increase according to the MOI but in a non-significant way compared to the GF condition. These results consolidate the hypothesis that the addition of the $L f$ CECT5716 in the co-cultured model of Caco2 cells does not change the metabolic pathway of dietary fat (Fig. 4). As expected, there was no statistical difference of $\mathrm{U}-{ }^{13} \mathrm{C}$ oleic acid content in the apical chamber here (data not shown).

\section{DISCUSSION}

We conducted the first study evaluating the capacity of $L f$ CECT5716 isolated from healthy mother's breast milk, to restore juvenile growth and maturation time in Drosophila in a condition of undernutrition. By using a simple preclinical screening, we showed that $L f$ CECT5716 has a significant impact on juvenile growth and maturation time upon undernutrition and that this beneficial effect was conserved when $L f$ CECT5716 was added to an infant formula in appropriate amounts $\left(10^{8}\right.$ CFU). Finally, we showed in vitro that this growth-promoting effect was probably not mediated by an increased uptake of lipid by the enterocytes. As mentioned in a previous study, all macronutrients could be good candidate to explain the phenotype induced by the Lf CECT5716 since Lactobacillus association are able to induce a host transcriptome response restricted to the midgut of drosophila. ${ }^{21}$ But we've chosen the lipids for two reasons. Storelli et al, shown that the enrichment of the low protein diet with sugar didn't promote growth. ${ }^{9}$ In the same paper, the role of protein and amino acids was extensively studied. The growth phenotype was mediated trough the TOR activity whose diet-derived branched amino-acids are the main activator. The hypothesis of an increase in amino-acids intake was reinforced by two recent publications showing the properties of the Lactobacillus to induce the peptidase activity in the Drosophila's gut trough complex bacterial 


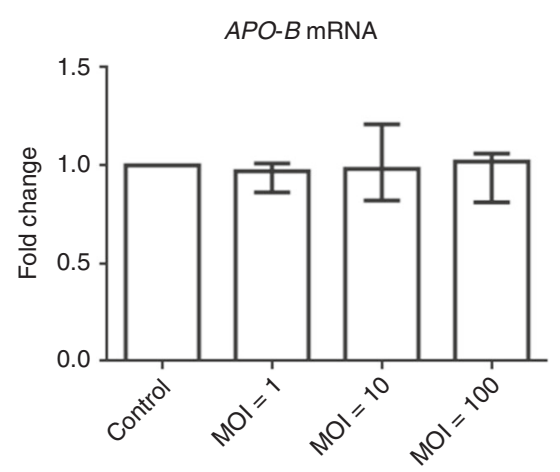

Bacterial condition

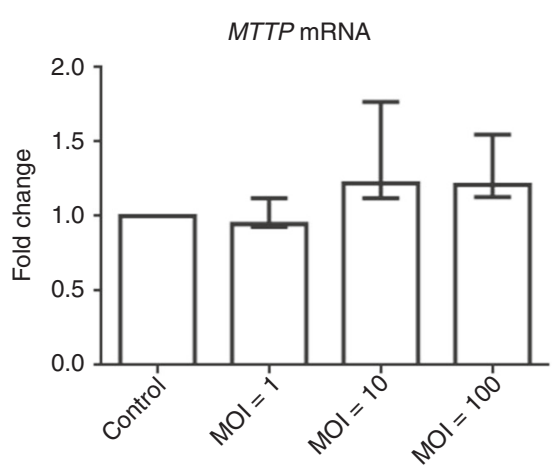

Bacterial condition

SAR1B mRNA

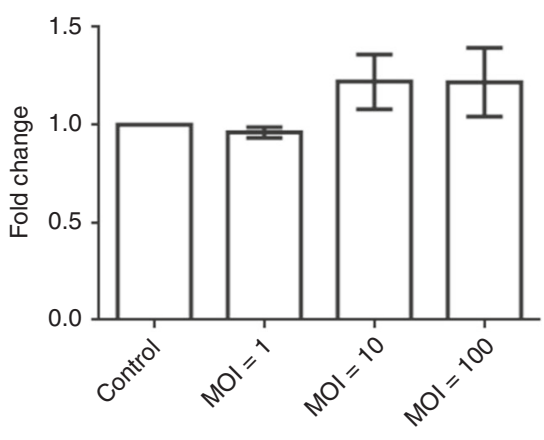

Bacterial condition

Fig. 4 Impact of $\mathbf{L f}$ CECT5716 on regulation of the main genes involved chylomicron synthesis in Caco2/TC7 cells. After $6 \mathrm{~h}$ of

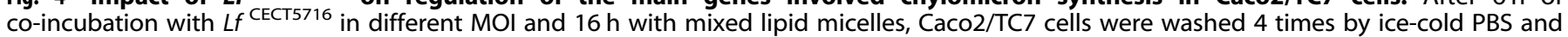
harvested. Total RNA was extract, reverse transcription was performed, and quantitative PCR was done using specific primers for $A P O-B, S A R 1 B$, and MTTP genes. Experiment was done three times in triplicate.

cell wall sensing machinery. ${ }^{22,23}$ That's why the study of the regulation of lipid intakes by the Lf CECT5716 is a new original issue that has not been explored before.

We have found that the intensity of the $L f^{\text {CECT5716 }}$ growthpromoting effect seems to be species dependent. In the drosophila model, previous studies also suggest different growth-promoting effects in different commensal bacteria. Storelli et al. have compared strains from two different commensal bacteria species in the same animal model: $L$. plantarum and Enterococcus faecalis. ${ }^{9}$ Contrary to the $L$. plantarum strains, E. faecalis strains did not promote juvenile growth. This shows that all strains issued from a gut microbiota do not have the same functionality to provide a growthpromoting effect like $L$. plantarum. In our work, we have shown that $L f$ CECT5716 is a qualified strain for a growth-promoting application as a member of the Lactobacillus family. This confirms that growth-promoting functionalities can be found in nonplantarum Lactobacillus species, as previously observed (ref. patent: WO2015173386A1).

The functionality of $L f^{\text {CECT5716 }}$ is probably more complex than a simple power attributed to one bacterial species. Our results found a statistical difference between the promoting effects of the two control strains of $L$. plantarum ( $L p^{\mathrm{NIZO} 2877}$ and $\left.L p^{\mathrm{WJL}}\right)$ but no statistical difference between $L f^{\text {CECT5716 }}$ and $L p^{\text {NIZO2877 }}$ despite their belonging to two different Lactobacillus species. Storelli et al. found a statistical difference in the effect on developmental timing of two $L$. plantarum strains $\left(L p{ }^{\text {WJL }}\right.$ and $L p$ IBDML1 $){ }^{9}$ Schwarzer et al. found a statistical difference in the growthpromoting effect of the same $L p$ WJL and $L p$ NIZO2877 in the drosophila model and in the mouse model. ${ }^{6}$ All these results demonstrate the important functional specificity among strains for the same species. Recently, Leulier's laboratory screened the growth-promoting effect of 200 different L. plantarum strains (data not published) and classified the strains into four categories related to the effect of control conditions:

- Strains with no effect, statistically identical to the GF condition. - Strains with an intermediate effect, statistically higher than the GF condition but statistically lower than the $L p{ }^{\text {WJL }}$ control (e.g.: Lp ${ }^{\text {NIZO2877). }}$

- Strain with a marked effect, statistically identical to the effect of the control strain $L p$ WJL.

- Strains with a strong effect, statistically higher than the effect of the control strain $L p^{\mathrm{WL}}$ (e.g.: $\left.L p^{\mathrm{G} 821}\right)$.

Yet, to our knowledge, $L f$ CECT5716 issued from human breast milk is only the fourth $L$. fermentum strain qualified for its growthpromoting effect in drosophila as part of the screening carried out for the patent (data not published). Regarding growth promotion, two strains were qualified as having a marked effect on growth ( $L f$ ${ }_{\text {ATCC9338 }}$ and $L f^{\text {ATCC14931 }}$ ) and one strain as having no effect $\left(L f^{\mathrm{KLD}}\right)$. Regarding maturation time, $L f f^{\text {ATCC9338 }}$ and $L f$ ATCC14931 were qualified as intermediate and $L f{ }^{\mathrm{KLD}}$ did not have any effect. $L f$ CECT5716 can be considered as an intermediate strain according to its growth-promoting effect (compared to $L p^{\mathrm{WJL}}$ ), but in the same manner as $L$. plantarum, different effects could likely co-exist for other $L$. fermentum species.

The growth-promoting effect seems to be dependent on the concentration of the bacterial inoculums. Two different concentrations of $L f{ }^{C E C T 5716}$ were tested in the same infant formula: $10^{4}$ CFU in the HMF-LF4 (ready-to-use industrial formula) and $10^{8} \mathrm{CFU}$ in the HMF-LF8 (homemade formula) both being of the same composition for macronutrients. Moreover, the HMF did not have any effect on larval growth. A rational of this dose-effect comes from the possible cellular mechanism by which $L f^{\text {CECT5716 }}$ sustains juvenile growth. The drosophila microbiota is able to impact the 
transcriptome of the mid-gut and to induce the expression of several genes involved in host metabolism such as proteases, lipases, and phosphatases. ${ }^{21}$ For the growth-promoting effect, L. plantarum is able to induce the transcription of host proteases and activate a TOR-dependent nutrient-sensing amino acidmediated pathway., ${ }^{9,24}$ This induction leads to the secretion of ecdysone (a drosophila steroid hormone) and insulin-like peptides, which promote juvenile growth. We can hypothesize that this pathway could have a dose-trigger to be functional. Several transcriptional pathways induced by the microbiota have already been shown to be concentration dependent in other models, such as mice. Lactobacillus casei $32 \mathrm{G}$ induces a change in the ecologic profile of the gut microbiota in the mice cecum, which is associated with changes in the immune response in a concentrationdependent manner. ${ }^{23}$ Lactobacillus brevis OPK-3 inhibits adipogenesis by downregulation of adiponectin as leptin or transcriptional factor as peroxisome proliferator-activated receptor gamma and liver $X$ receptor alpha. ${ }^{25}$ These downregulations were systematically influenced by the probiotic concentration.

The second part of our work evaluating the mechanistic aspects do not support a significant effect of $L f^{\text {CECT5716 }}$ on lipid uptake by

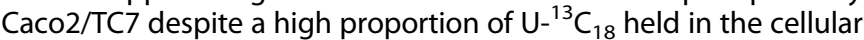
compartment. CMs are the main form of lipoprotein secreted by intestinal cells for the transport of dietary fat. ${ }^{26} \mathrm{CM}$ synthesis is a complex mechanism involving re-esterification and translocation of cellular lipid pool from lipolytic products. The CM synthesis involved three crucial main proteins known to be upregulated during the fed state: Apo-B48, microsomal triglyceride transport protein (MTTP), and Sar-1 GTPase. The lack of transcription regulation of the corresponding genes $A P O B, M T T P$, and SAR1B further sustain the hypothesis that $L f$ CECT5716 is not able to increase the synthesis of CM in Caco2/TC7 cells.

Finally, an important result of our study is the preservation of the growth-promoting effect of the $L f$ CECT5716 when added in a commercial infant formula. We bring here the first proof of concept of an enriched infant formula with a strain isolated from breast milk, which has a superior growth-promoting effect compared to a standard formula in stunting conditions. During the first days of life, the beneficial effect of the breast feeding has been widely described. Moreover, the breast milk microbiome seems to play an important role in the acquisition of the newborn immunity and in infection and metabolic disease prevention. ${ }^{27}$ In this context, medical applications of $L f{ }^{\text {CECT5716 }}$ for use in malnutrition could be relevant. Actually, few clinical trials have studied the impact of probiotics in infant formula on infant growth without effect. ${ }^{15,28}$ However, trials have been made only in healthy children, and as we showed here, the promoting effect is maximal upon undernutrition. Trials in an undernourished pediatric population are needed to confirm our results in the animal.

In conclusion, the bacteria Lf CECT5716 isolated from human breast milk qualified as an intermediate growth and maturationpromoting strain upon undernutrition in a drosophila model. This effect seems to be unrelated to an improvement in lipid absorption. The growth-promoting effect of $L f$ CECT5716 was maintained when $L f$ CECT5716 was added to an infant formula in an appropriate concentration.

\section{ACKNOWLEDGEMENTS}

P.P. received research support as a second-year master's student from LACTALIS NUTRITION SANTE ${ }^{\oplus}$ France. P.P would like to thank Gwenael Jan from the French National Institute for Agriculture, Food and Environment (UMR STLO, Rennes, France) for his precious help in developing the cell co-culture model.

\section{AUTHOR CONTRIBUTIONS}

P.P., N.P. and F.L. have contributed to the conception and design of the study. P.P., A.P., M.C.M., E.M., V.S., L.M., and C.L.-P have contributed to acquisition of data. All authors have contributed to the analysis and interpretation of data. P.P. drafted the article. All authors reviewed and revised it critically for intellectual content and gave their final approval of the version to be published. P.P. would like to thanks Gwenaël Jan for his precious advice in cell and bacteria co-culture.

\section{ADDITIONAL INFORMATION}

The online version of this article (https://doi.org/10.1038/s41390-020-0774-0) contains supplementary material, which is available to authorized users.

Competing interests: The authors declare no competing interests.

Publisher's note Springer Nature remains neutral with regard to jurisdictional claims in published maps and institutional affiliations.

\section{REFERENCES}

1. Horton, R. \& Lo, S. Nutrition: a quintessential sustainable development goal. Lancet 382, 371-372 (2013).

2. Kyle, U. G., Shekerdemian, L. S. \& Coss-Bu, J. A. Growth failure and nutrition considerations in chronic childhood wasting diseases. Nutr. Clin. Pract. 30, 227-238 (2015).

3. Fazeli, P. K. \& Klibanski, A. Determinants of growth hormone resistance in malnutrition. J. Endocrinol. 220, R57-R65 (2014).

4. Subramanian, $\mathrm{S}$. et al. Persistent gut microbiota immaturity in malnourished Bangladeshi children. Nature 510, 417-421 (2014).

5. Charbonneau, M. R. et al. Sialylated milk oligosaccharides promote microbiotadependent growth in models of infant undernutrition. Cell 164, 859-871 (2016).

6. Schwarzer, M. et al. Lactobacillus plantarum strain maintains growth of infant mice during chronic undernutrition. Science 351, 854-857 (2016).

7. Smith, M. I. et al. Gut microbiomes of Malawian twin pairs discordant for kwashiorkor. Science 339, 548-554 (2013).

8. Blanton, L. V. et al. Gut bacteria that prevent growth impairments transmitted by microbiota from malnourished children. Science 351, aad3311 (2016).

9. Storelli, G. et al. Lactobacillus plantarum promotes Drosophila systemic growth by modulating hormonal signals through TOR-dependent nutrient sensing. Cell Metab. 14, 403-414 (2011).

10. Erkosar, B. \& Leulier, F. Transient adult microbiota, gut homeostasis and longevity: novel insights from the Drosophila model. FEBS Lett. 588, 4250-4257 (2014).

11. Matos, R. C. \& Leulier, F. Lactobacilli-host mutualism: "learning on the fly". Microb. Cell Fact. 13, S6 (2014)

12. Martín, R. et al. Probiotic potential of 3 Lactobacilli strains isolated from breast milk. J. Hum. Lact. 21, 8-17 (2005). quiz 18-21, 41.

13. Pérez-Cano, F. J., Dong, H. \& Yaqoob, P. In vitro immunomodulatory activity of Lactobacillus fermentum CECT5716 and Lactobacillus salivarius CECT5713: two probiotic strains isolated from human breast milk. Immunobiology 215, 996-1004 (2010).

14. Maldonado, J. et al. Human milk probiotic Lactobacillus fermentum CECT5716 reduces the incidence of gastrointestinal and upper respiratory tract infections in infants. J. Pediatr. Gastroenterol. Nutr. 54, 55-61 (2012).

15. Maldonado-Lobón, J. A. et al. Long-term safety of early consumption of Lactobacillus fermentum CECT5716: a 3-year follow-up of a randomized controlled trial. Pharmacol. Res. 95-96, 12-19 (2015).

16. Chateau, D. et al. Lipid micelles stimulate the secretion of triglyceride-enriched apolipoprotein B48-containing lipoproteins by Caco-2 cells. J. Cell. Physiol. 202, 767-776 (2005).

17. Levy, E., Mehran, M. \& Seidman, E. Caco-2 cells as a model for intestinal lipoprotein synthesis and secretion. FASEB J. 9, 626-635 (1995).

18. Kim, E.-K., Park, Y. M., Lee, O. Y. \& Lee, W.-J. Draft genome sequence of Lactobacillus plantarum strain WJL, a Drosophila gut symbiont. Genome Announc. https://doi.org/10.1128/genomeA.00937-13 (2013).

19. Vors, C. et al. Coupling in vitro gastrointestinal lipolysis and Caco-2 cell cultures for testing the absorption of different food emulsions. Food Funct. 3, 537-546 (2012).

20. Gabert, L. et al. $13 \mathrm{C}$ tracer recovery in human stools after digestion of a fat-rich meal labelled with [1,1,1-13C3]tripalmitin and [1,1,1-13C3]triolein. Rapid Commun. Mass Spectrom. 25, 2697-2703 (2011).

21. Erkosar, B. et al. Drosophila microbiota modulates host metabolic gene expression via IMD/NF-KB signaling. PLOS ONE 9, e94729 (2014).

22. Matos, R. C. et al. D-Alanylation of teichoic acids contributes to Lactobacillus plantarum-mediated Drosophila growth during chronic undernutrition. Nature Microbiology 2, 1635-1647 (2017)

23. Aktas, B. et al. The effect of Lactobacillus casei $32 \mathrm{G}$ on the mouse cecum microbiota and innate immune response is dose and time dependent. PLOS ONE 10, e0145784 (2015)

24. Erkosar, B. et al. Pathogen virulence impedes mutualist-mediated enhancement of host juvenile growth via inhibition of protein digestion. Cell Host Microbe 18, 445-455 (2015). 
Probiotic from human breast milk, Lactobacillus fermentum, promotes...

$\mathrm{P}$ Poinsot et al.

25. Park, J.-E., Oh, S.-H. \& Cha, Y.-S. Lactobacillus brevis OPK-3 isolated from kimchi inhibits adipogenesis and exerts anti-inflammation in 3T3-L1 adipocyte. J. Sci. Food Agric. 94, 2514-2520 (2014).

26. Levy, E. Insights from human congenital disorders of intestinal lipid metabolism. J. Lipid Res. 56, 945-962 (2015).
27. Ojo-Okunola, A., Nicol, M. \& du Toit, E. Human breast milk bacteriome in health and disease. Nutrients 10, 1643 (2018).

28. Braegger, C. et al. Supplementation of infant formula with probiotics and/or prebiotics: a systematic review and comment by the ESPGHAN Committee on Nutrition. J. Pediatr. Gastroenterol. Nutr. 52, 238-250 (2011). 